\title{
PERFIL EPIDEMIOLÓGICO E ATUAÇÃO FISIOTERÁPICA EM PACIENTES HOSPITALIZADOS- ANÁLISE RESTROSPECTIVA
}

Débora Mayumi de Oliveira Kawakami, Junior Francisco da Silva, Natália das Neves Andrade, Isabella Maria Gonçalves Silva, Camila Aparecida Gibim, Anne Kastelianne França da Silva, Susimary Aparecida Trevizan Padulla

Universidade Estadual Paulista "Júlio de Mesquita Filho" - UNESP, Departamento de Fisioterapia, Curso de Fisioterapia, Presidente Prudente, São Paulo. E-mail: debora mayumi@hotmaill.com

\section{RESUMO}

A prevalência de doenças crônico-degenerativas e expectativa de vida tem aumentado, o que está associado ao maior número de internações entre os idosos. Nesse sentido, a fisitoterapia é uma alternativa de tratamento nessa população e possui diversas alternativas de reabilitação com o intuito de favorecer a funcionalidade do paciente. O objetivo deste estudo foi caracterizar o perfil dos pacientes internados e apontar as técnicas fisioterápicas utilizadas. Este estudo tem caráter retrospectivo, com análise de prontuários com prescrição de fisioterapia de um hospital público no período de 2015 a 2017. Observou-se que a maioria dos pacientes são homens, idosos, com HAS ou mais de um fator de risco, tabagistas/ex-tabagistas, etilistas, com IMC normal, tempo médio de internação hospitalar de 8 a 19 dias e diagnóstico na especialidade oncologia. As técnicas mais utilizadas são padrão ventilatório, reeducação diafragmática, mobilização ativa e deambulação. As técnicas empregadas com maior frequência dependem da participação ativa do paciente, o que nos permite concluir a importância do fisioterapeuta em unidades hospitalares, dado o seu perfil incentivador à promoção de atividades que possam reestabelecer a funcionalidade dos pacientes.

Palavras-chave: Perfil epidemiológico, pacientes hospitalizados, fisioterapia respiratória, mobilização precoce.

\section{EPIDEMIOLOGICAL PROFILE AND PHYSIOTHERAPY IN HOSPITALIZED PATIENTS - RESTROSPECTIVE ANALYSIS}

\begin{abstract}
The prevalence of chronic degenerative diseases and life expectancy has increased, which is associated with the higher number of hospitalizations among the elderly. In this sense, physical therapy is an alternative treatment in this population and has several rehabilitation alternatives in order to favor the patient's functionality. The objective of this study was to characterize the profile of hospitalized patients and to point out the physiotherapeutic techniques used. This study is retrospective, with an analysis of records with physical therapy prescription of a public hospital in the period from 2015 to 2017. It was observed that the majority of the patients are men, elderly, with hypertension or more than one risk factor, smokers/ex-smokers, alcoholics, with normal BMI, mean hospital stay of 8 to 19 days and diagnosis in specialty oncology. The most used techniques are exercises breathing, diaphragmatic reeducation, active mobilization and walking. The most frequently used techniques depend on the active participation of the patient, which allows us to conclude the importance of the physiotherapist in hospital units, given their incentive profile to promote activities that can reestablish the patients' functionality.
\end{abstract}

Keywords: Epidemiological profile, hospitalized patients, respiratory physiotherapy, early mobilization. 


\section{INTRODUÇÃO}

$\mathrm{Na}$ esfera hospitalar, muito tem se debatido sobre a composição do sistema de saúde pública e a gestão deste setor quando se refere à internação hospitalar. A organização do sistema de saúde vem sendo aperfeiçoada a todo momento, porém, atualmente é observada uma superlotação que se inicia nos serviços de emergência e que acarreta inúmeras internações hospitalares. ${ }^{[1]}$

De 2015 a 2017 no Brasil, houve registro de em média 34 milhões de internações hospitalares pelo Sistema Único de Saúde (SUS) gerando gasto de aproximadamente 33 bilhões de reais. A região Sudeste foi a que apresentou maior taxa de gastos com pacientes internados, sendo 14 bilhões de reais com aproximadamente 13 milhões de internações hospitalares. ${ }^{\text {[2] }}$

Diversos hospitais, brasileiros e de outros países, exibem cenários de falta de leitos com taxa de ocupação superior a $100 \%{ }^{[1]}$. Alguns autores justificam que essa superlotação corresponde à taxa crescente da expectativa de vida e à inversão da pirâmide etária, marcada pelo envelhecimento populacional e pela presença de doenças crônico degenerativas que envolvem cuidados contínuos e muitas vezes de alto custo. ${ }^{[3]}$

Um aspecto importante são as comorbidades preexistentes à internação, sendo as mais comuns, na população em geral, o diabetes mellitus e a hipertensão arterial sistêmica. Muitos pacientes internados por disporem de quadro clínico debilitado podem apresentar declínio funcional agravado pela associação à presença de fatores de risco, ocasionando pior prognóstico e maior tempo de internação hospitalar. ${ }^{[4]}$

Dessa forma, a fisioterapia tem como objetivo atuar no restabelecimento ou manutenção da funcionalidade física e respiratória do paciente, sobretudo empregando um leque de técnicas de fisioterapia respiratória e motora aplicadas em muitas especialidades dentro do ambiente hospitalar. ${ }^{[5]}$

Em virtude da existência de muitas técnicas fisioterapêuticas, em alguns estudos foram observados heterogeneidade em tratamentos realizados pelos fisioterapeutas de outros países ${ }^{[6,7]}$. Sendo assim, esta pesquisa tem por objetivo traçar o perfil de pacientes internados em um hospital público de Presidente Prudente no período de 2015 a 2017 e que foram atendidos pelo serviço de fisioterapia de uma universidade, além de correlacionar com as técnicas fisioterapêuticas mais utilizadas de acordo com a especialidade atendida.

\section{METODOLOGIA}

\section{Desenho do estudo e população}

Para a construção desse estudo, foi realizada a avaliação retrospectiva de caráter descritiva, de prontuários de pacientes internados em duas clínicas/enfermarias do Sistema Único de Saúde de um hospital da cidade de Presidente Prudente/SP no período de 2015 a 2017 que tinham prescrição de fisioterapia. O estudo foi desenvolvido de acordo com os preceitos da Resolução 196/96, tendo sido aprovado pelo Comitê de Ética da Santa Casa e pelo Comitê de Ética em Pesquisa da Faculdade de Ciências e Tecnologia- FCT/Unesp (Proc. no 02592612.8.0000.5402) obedecendo a Resolução 466/2012 do Conselho Nacional de Saúde de 12/12/2012.

O serviço de fisioterapia fornecido pela Universidade é uma parceria do Serviço Integrado de Fisioterapia com o Departamento de Fisioterapia da Faculdade de Ciências e Tecnologia da Universidade Estadual Paulista - Fct/Unesp.

Foram incluídos 602 prontuários de pacientes, que foram submetidos a avaliação por meio de ficha pré-determinada do setor que, associada a evolução diária, compõe o prontuário fisioterapêutico. Os prontuários que não exibiam adequado preenchimento dos campos essenciais, como tratamento fisioterapêutico, foram desconsiderados da amostra. 


\section{Desfechos avaliados}

Foram extraídos dos prontuários:

1. Dados demográficos: Sexo, idade

2. Fatores de risco cardiovasculares: Hipertensão arterial, diabetes mellitus, tabagismo, dislipidemia, obesidade.

3. Aspectos relacionados a hospitalização: tempo de internação, ano de internação, especialidade (Cardiologia, Neurologia, Oncologia, Pneumologia, Ortopedia, Ginecologia/Nefrologia, Gastroenterologia e outros) e diagnóstico médico.

4. Tratamento fisioterapêutico realizado: fisioterapia respiratória (técnicas utilizadas como: vibrocompressão, inaloterapia, tapotagem, flutter, tosse, aspiração, padrão ventilatório, inspirômetro incentivador, respiração diafragmática, compressão, oxigenoterapia) e fisioterapia motora (passiva, ativa, ativa-assistida, resistida e deambulação).

\section{Análise dos dados}

Os dados foram extraídos por cinco avaliadores e organizados em uma planilha utilizando o programa Microsoft Office Excel 2007 para filtragem manual dos dados a serem empregados neste estudo. Após a filtragem manual dos dados, a análise estatística foi realizada a partir do Software da IBM SPSS Statistics 20 for Windows 7, utilizando-se a estatística descritiva em frequência (\%), média (desvio padrão) e dados de referência cruzada.

\section{RESULTADOS}

Entre o período de janeiro de 2015 a dezembro de 2017, 607 pacientes obtiveram prescrição de fisioterapia. Desse total de pacientes, 05 pacientes foram excluídos da pesquisa por apresentar prontuários incompletos.

Os 602 pacientes incluídos, foram discriminados de acordo com as especialidades de suas patologias, sendo: cardiologia, neurologia, oncologia, pneumologia, ortopedia, ginecologia/nefrologia, gastroenterologia e outros. Para caracterização da amostra, foram obtidos dados como idade, sexo, IMC e tempo de internação hospitalar, como mostra a tabela 
Tabela 1. Características da amostra

\begin{tabular}{|c|c|c|c|c|c|c|c|c|}
\hline & $\begin{array}{l}\text { Cardio } \\
\text { (n= } \\
92)\end{array}$ & $\begin{array}{l}\text { Neuro } \\
(n=42)\end{array}$ & $\begin{array}{l}\begin{array}{l}\text { Onco } \\
(n= \\
180)\end{array}\end{array}$ & $\begin{array}{l}\text { Pneu } \\
\text { mo } \\
(n=76)\end{array}$ & $\begin{array}{l}\text { Orto } \\
(n= \\
77)\end{array}$ & $\begin{array}{l}\text { Gineco/ } \\
\text { Nefro } \\
(n=12)\end{array}$ & $\begin{array}{l}\text { Gast } \\
\text { ro } \\
(n= \\
100)\end{array}$ & 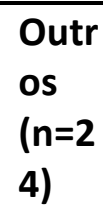 \\
\hline Idade média (DP) & $\begin{array}{l}65,4 \\
(12,4)\end{array}$ & $\begin{array}{l}64,55 \\
(17,7)\end{array}$ & $\begin{array}{l}63,9 \\
(12,7)\end{array}$ & $\begin{array}{l}67,7 \\
(17,3)\end{array}$ & $\begin{array}{l}65,0 \\
(16,9)\end{array}$ & $\begin{array}{l}61,4 \\
(11,8)\end{array}$ & $\begin{array}{l}59,1 \\
(14, \\
9)\end{array}$ & $\begin{array}{l}61,2 \\
(20,0 \\
)\end{array}$ \\
\hline \multicolumn{9}{|l|}{ Sexo n (\%) } \\
\hline Feminino & $\begin{array}{l}28 \\
(30,4 \\
\%)\end{array}$ & $\begin{array}{l}15 \\
(35,7 \% \\
)\end{array}$ & $\begin{array}{l}65 \\
(36,1 \% \\
)\end{array}$ & $\begin{array}{l}34 \\
(44,7 \\
\%)\end{array}$ & $\begin{array}{l}42 \\
(54,5 \\
\%)\end{array}$ & $\begin{array}{l}6 \\
(50,0 \%)\end{array}$ & $\begin{array}{l}61 \\
(61, \\
0 \%)\end{array}$ & $\begin{array}{l}13 \\
(54,2 \\
\%)\end{array}$ \\
\hline Masculino & $\begin{array}{l}64 \\
(69,6 \\
\%)\end{array}$ & $\begin{array}{l}27 \\
(64,3 \% \\
)\end{array}$ & $\begin{array}{l}115 \\
(63,9 \% \\
)\end{array}$ & $\begin{array}{l}42 \\
(55,3 \\
\%)\end{array}$ & $\begin{array}{l}35 \\
(45,5 \\
\%)\end{array}$ & $\begin{array}{l}6 \\
(50,0 \%)\end{array}$ & $\begin{array}{l}39 \\
(39, \\
0 \%)\end{array}$ & $\begin{array}{l}11 \\
(45,8 \\
\%)\end{array}$ \\
\hline Prescrição n (\%) & $\begin{array}{l}91 \\
(15,1 \\
\%)\end{array}$ & $\begin{array}{l}42 \\
(7 \%)\end{array}$ & $\begin{array}{l}180 \\
(29,9 \%\end{array}$ & $\begin{array}{l}76 \\
(12,6 \\
\%)\end{array}$ & $\begin{array}{l}77 \\
(12,8 \\
\%)\end{array}$ & $\begin{array}{l}12 \\
(2,0 \%)\end{array}$ & $\begin{array}{l}100 \\
(16, \\
6 \%)\end{array}$ & $\begin{array}{l}24 \\
(4 \%)\end{array}$ \\
\hline \multicolumn{9}{|l|}{ IMC (\%) } \\
\hline Abaixo do peso & $\begin{array}{l}1 \\
(1,1 \%)\end{array}$ & )$^{1(2,4 \%}$ & $\begin{array}{l}7 \\
(3,9 \%)\end{array}$ & $\begin{array}{l}5 \\
(6,6 \%)\end{array}$ & $\begin{array}{l}1(1,3 \\
\%)\end{array}$ & $0(0 \%)$ & $\begin{array}{l}4 \\
(4 \%)\end{array}$ & $\begin{array}{l}5(20, \\
8 \%)\end{array}$ \\
\hline Normal & $\begin{array}{l}43 \\
(47,3 \\
\%)\end{array}$ & $\begin{array}{l}18 \\
(42,9 \% \\
)\end{array}$ & $\begin{array}{l}89 \\
(49,4 \% \\
)\end{array}$ & $\begin{array}{l}37 \\
(48,7 \\
\%)\end{array}$ & $\begin{array}{l}36 \\
(46,8 \\
\%)\end{array}$ & $\begin{array}{l}5 \\
(41,7 \%)\end{array}$ & $\begin{array}{l}36 \\
(36 \\
\%)\end{array}$ & $\begin{array}{l}13 \\
(54,2 \\
\%)\end{array}$ \\
\hline Sobrepeso & $\begin{array}{l}15 \\
(16,5 \\
\%)\end{array}$ & $0(0 \%)$ & $\begin{array}{l}12 \\
(6,7 \%)\end{array}$ & $\begin{array}{l}4 \\
(5,3 \%)\end{array}$ & $0(0 \%)$ & $0(0 \%)$ & $\begin{array}{l}0 \\
(0 \%)\end{array}$ & $\begin{array}{l}0 \\
(0 \%)\end{array}$ \\
\hline Obesidade & $\begin{array}{l}0 \\
(0 \%)\end{array}$ & $\begin{array}{l}7 \\
(16,7 \% \\
)\end{array}$ & $\begin{array}{l}0 \\
(0 \%)\end{array}$ & $\begin{array}{l}3 \\
(3,9 \%)\end{array}$ & $\begin{array}{l}6 \\
(7,8 \%)\end{array}$ & $\begin{array}{l}3 \\
(25 \%)\end{array}$ & $\begin{array}{l}23 \\
(23 \\
\%)\end{array}$ & $\begin{array}{l}4 \\
(16,7 \\
\%)\end{array}$ \\
\hline $\begin{array}{l}\text { Tempo de internação } \\
\text { Hospitalar média (DP) }\end{array}$ & $\begin{array}{l}13,8 \\
(18,3)\end{array}$ & $\begin{array}{l}11,2 \\
(8,2)\end{array}$ & $\begin{array}{l}12,7 \\
(12,0)\end{array}$ & $\begin{array}{l}19,0 \\
(30,4)\end{array}$ & $\begin{array}{l}12,3 \\
(7,2)\end{array}$ & $8,3(4,2)$ & $\begin{array}{l}11,3 \\
(7,9)\end{array}$ & $\begin{array}{l}10,5 \\
(5,4)\end{array}$ \\
\hline
\end{tabular}

Legenda: $\mathrm{n}=$ tamanho da amostra; DP= desvio padrão; IMC= Índice de massa corpórea; Cardio= Cardiologia; Neuro= Neurologia; Onco= Oncologia; Pneumo= Pneumologia; Orto= Ortopedia; Gineco/Nefro= Ginecologia/Nefrologia; Gastro= Gastroenterologia.

Em relação aos fatores de risco, os mais prevalentes foram a Hipertensão Arterial Sistêmica $47(47,5 \%)$ e Dislipidemia $11(11,1 \%)$ no grupo oncológico, o Diabetes Mellitus no grupo de doenças gastrointestinais $33(33 \%)$ e pacientes com presença de mais de um dos fatores de risco mencionado sendo oncológicos e cardiopatas, ambos com 33 (33,3\%). Os dados estão apontados na Tabela 2, assim como $\mathrm{n}(\%)$ de pacientes em relação ao tabagismo e etilismo. 
Tabela 2. Prevalência dos fatores de risco, estratificado por especialidade.

\begin{tabular}{|c|c|c|c|c|c|c|c|}
\hline $\begin{array}{l}\text { Cardio } \\
(n=92)\end{array}$ & $\begin{array}{l}\text { Neuro } \\
(n=42)\end{array}$ & $\begin{array}{l}\text { Onco } \\
(n=180)\end{array}$ & $\begin{array}{l}\text { Pneum } \\
\text { o } \\
(n=76)\end{array}$ & $\begin{array}{l}\text { Orto } \\
(n=77)\end{array}$ & $\begin{array}{l}\text { Gineco/N } \\
\text { efro } \\
(n=12)\end{array}$ & $\begin{array}{l}\text { Gastr } \\
0 \\
(n= \\
100)\end{array}$ & $\begin{array}{l}\text { Outr } \\
\text { os } \\
\text { (n=24 } \\
\text { ) }\end{array}$ \\
\hline
\end{tabular}

Fatores de Risco

$\mathrm{n}(\%)$

HAS

$\begin{array}{llllllll}28 & 13 & 47 & 6 & 20 & 6 & 42 & 6 \\ (40,6 \%) & (31 \%) & (47,5 \%) & (26,1 \%) & (26 \%) & (50 \%) & (42 \%) & (35,3\end{array}$

\begin{tabular}{|c|c|c|c|c|c|c|c|c|}
\hline DM & $\begin{array}{l}3 \\
(4,3 \%)\end{array}$ & $\begin{array}{l}1 \\
(2,4 \%)\end{array}$ & $\begin{array}{l}8 \\
(8,1 \%)\end{array}$ & $\begin{array}{l}0 \\
(0 \%)\end{array}$ & $\begin{array}{l}1 \\
(1,3 \%)\end{array}$ & $1(8,3 \%)$ & $\begin{array}{l}33 \\
(33 \%)\end{array}$ & $\begin{array}{l}2 \\
(11,8 \\
\%)\end{array}$ \\
\hline DLP & $\begin{array}{l}5 \\
(7,2 \%)\end{array}$ & $\begin{array}{l}1 \\
(2,4 \%)\end{array}$ & $\begin{array}{l}11 \\
(11,1 \%)\end{array}$ & $\begin{array}{l}1 \\
(4,3 \%)\end{array}$ & $\begin{array}{l}3 \\
(3,9 \%)\end{array}$ & $\begin{array}{l}0 \\
(0 \%)\end{array}$ & $\begin{array}{l}4 \\
(4 \%)\end{array}$ & $\begin{array}{l}2 \\
(11,8 \\
\%)\end{array}$ \\
\hline$>1$ fator de risco & $\begin{array}{l}33 \\
(47,8 \%)\end{array}$ & $\begin{array}{l}8 \\
(19 \%)\end{array}$ & $\begin{array}{l}33 \\
(33,3 \%)\end{array}$ & $\begin{array}{l}16 \\
(69,6 \%)\end{array}$ & $\begin{array}{l}24 \\
(31,2 \%)\end{array}$ & $\begin{array}{l}3 \\
(25 \%)\end{array}$ & $\begin{array}{l}1 \\
(1 \%)\end{array}$ & $\begin{array}{l}5 \\
(29,4 \\
\%)\end{array}$ \\
\hline 0 fator de risco & $\begin{array}{l}0 \\
(0 \%)\end{array}$ & $\begin{array}{l}19 \\
(45,2 \%)\end{array}$ & $\begin{array}{l}0 \\
(0 \%)\end{array}$ & $\begin{array}{l}0 \\
(0 \%)\end{array}$ & $\begin{array}{l}29 \\
(37,7 \%)\end{array}$ & $2(16,7 \%)$ & $\begin{array}{l}20 \\
(20 \%)\end{array}$ & $\begin{array}{l}2 \\
(11,8 \\
\%)\end{array}$ \\
\hline
\end{tabular}

Tabagismo

Não fumante

$\begin{array}{llllllll}35 & 16 & 58 & 26 & 43 & & 58 & 12 \\ (39,3 \%) & (38,1 \%) & (32,4 \%) & (34,7 \%) & (55,8 \%) & 4(33,3 \%) & (59,2 & (50 \%) \\ \end{array}$

$\begin{array}{lllllllll}\text { Ex-Fumante } & 47 & 21 & 100 & 43 & 26 & 6 & 35 & 9 \\ & (52,8 \%) & (50 \%) & (55,9 \%) & (57,3 \%) & (33,8 \%) & (50 \%) & \begin{array}{l}(35,7 \\ \%)\end{array} & \begin{array}{l}(37,5 \\ \%)\end{array} \\ \text { Fumante } & 7 & 5 & 21 & 6 & 8 & & 5 & 3 \\ & (7,9 \%) & (11,9 \%) & (11,7 \%) & (8,0 \%) & (10,4) & 2(16,7 \%) & (5,1 \% & (12,5 \\ & & & & & & ) & \%)\end{array}$

\section{Etilismo}

Não

$\begin{array}{llllllll}52 & 26 & 119 & 59 & 53 & 6 & 63 & 15 \\ (58,4 \%) & (63,4 \%) & (68,8 \%) & (77,6 \%) & (69,7 \%) & (50 \%) & (64,3 & (62,5 \\ & & & & \%)\end{array}$

\begin{tabular}{lllllllll} 
Sim, 1x/mês & 19 & 7 & 15 & 7 & 5 & 3 & 16 & 7 \\
& $(21,3 \%)$ & $(17,1 \%)$ & $(8,7 \%)$ & $(9,2 \%)$ & $(6,6 \%)$ & $(25 \%)$ & $\begin{array}{l}(16,3 \\
\%)\end{array}$ & $\begin{array}{l}(29,2 \\
\%)\end{array}$ \\
Sim, 1x/semana & 8 & 5 & 21 & 4 & 8 & & 8 & 1 \\
& $(9 \%)$ & $(12,2 \%)$ & $(12,1 \%)$ & $(5,3 \%)$ & $(10,5 \%)$ & $1(8,3 \%)$ & $(8,2 \%$ & $(4,2 \%$ \\
Diariamente & 10 & 3 & 18 & 6 & 10 & & 1 & 1 \\
& $(11,2 \%)$ & $(7,3 \%)$ & $(10,4 \%)$ & $(7,9 \%)$ & $(13,2 \%)$ & $2(16,7 \%)$ & $(11,2$ & $(4,2 \%$ \\
& & & & & $\%)$ & 1 \\
\hline
\end{tabular}

Legenda: $n=$ tamanho da amostra; HAS= Hipertensão Arterial Sistêmica; DM= Diabetes Mellitus; DLP= Dislipdemia; Cardio= Cardiologia; Neuro= Neurologia; Onco= Oncologia; Pneumo= Pneumologia; Orto= Ortopedia; Gineco $/$ Nefro= Ginecologia/Nefrologia; Gastro= Gastroenterologia. 
O gráfico 1, apresenta dados relacionados as técnicas fisioterápicas utilizadas entre os anos de 2015 e 2017. Observa-se predomínio de padrão ventilatório e reeducação diafragmática, utilizados na fisioterapia respiratória, como apresentado no Gráfico 1, e de motora ativa e deambulação como evidenciado no Gráfico 2.

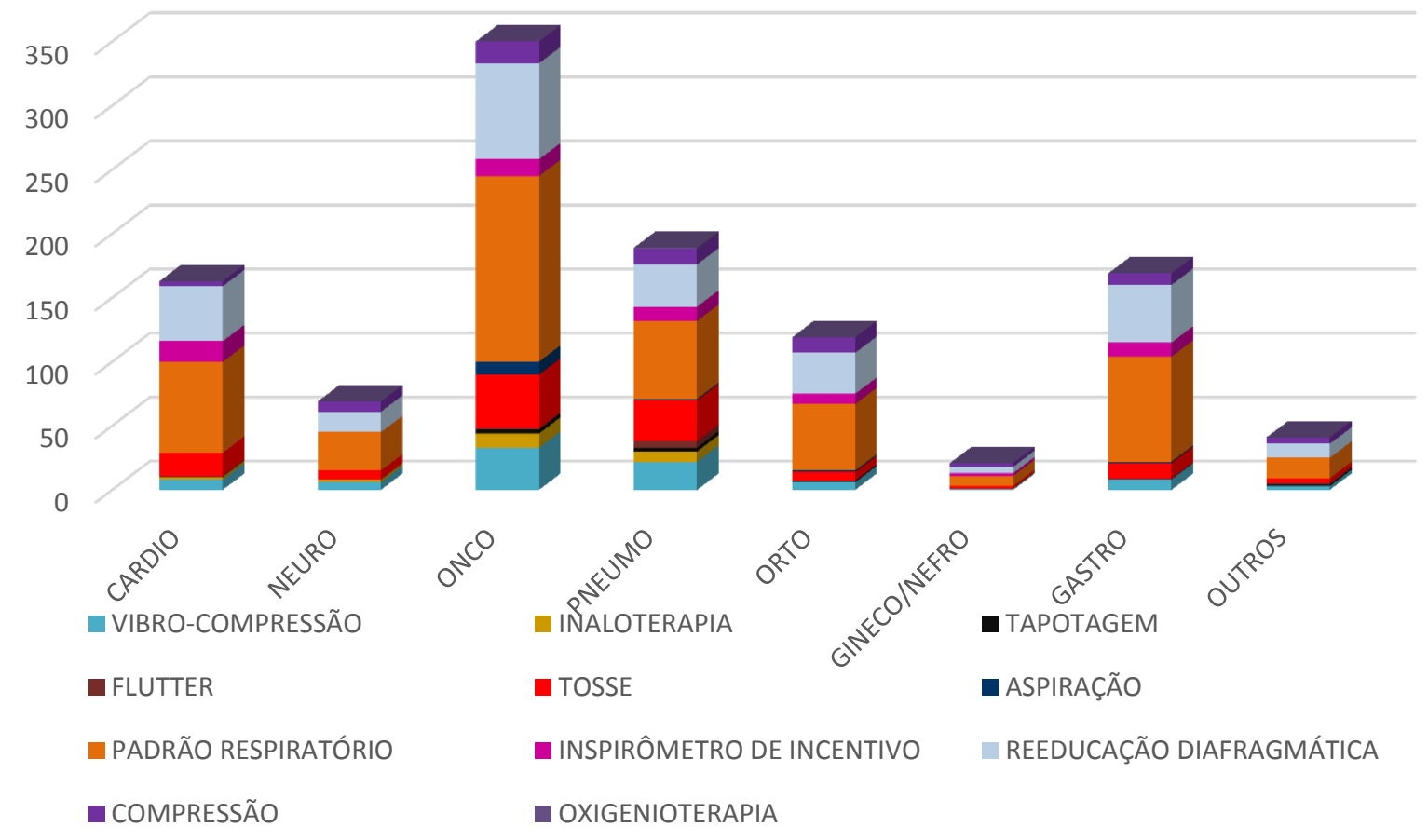

Gráfico 1. Distribuição de técnicas de fisioterapia respiratória de 2015 a 2017. 


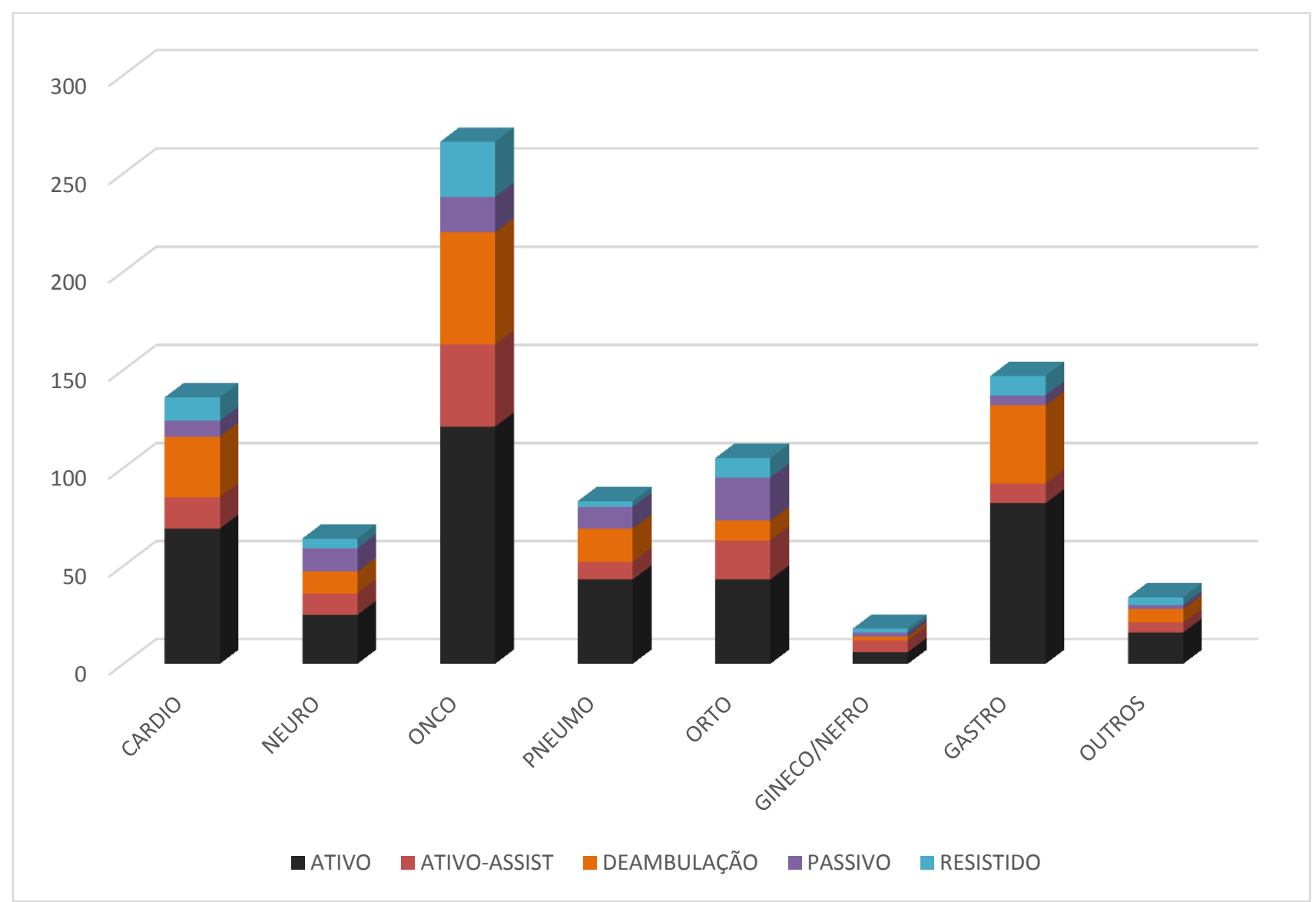

Gráfico 2. Distribuição de fisioterapia motora de 2015 a 2017.

\section{DISCUSSÃO}

De acordo com as análises, observou-se predomínio de pacientes do sexo masculino e com idade superior aos 60 anos. Os mesmos dados foram observados em estudo de Santos 2015, que relatou aproximadamente $60 \%$ da amostra com idade $>60$ anos e predomínio do sexo masculino em 55\%da amostra, sendo, o maior número de pacientes com prescrição de fisioterapia ainda na área de oncologia $(29,9 \%)$ e gastroenterologia $(16,6 \%)$.

No cenário atual, com o aumento da expectativa de vida e prevalência de doenças crônicodegenerativas, a alta taxa de hospitalização é mais frequente em pessoas idosas. Está relacionada a maior gravidade e pior prognóstico devido à maior debilidade fisiológica que também requer maior tempo de internação hospitalar. ${ }^{[8,9,10]}$

Segundo o DATASUS, o tempo médio de internação hospitalar possui variações de acordo com o sexo do paciente, nesse caso, homens ficariam mais tempo internados (6,8 dias) quando comparado as mulheres (4,6 dias). Em contrapartida, em nosso estudo, foi observado tempo médio para cada especialidade, variando de 8 a 19 dias de internação hospitalar. Sendo a amostra, composta por maioria dos pacientes com o IMC normal, com hipertensão arterial sistêmica ou presença de mais de um fator de risco (HAS, DM, DLP), tabagistas ou ex-tabagistas e etilistas/não etilistas em mesma proporção apesar de ambos não apresentaram diferenças estatisticamente significativas entre sobreviventes e não sobreviventes em internação. ${ }^{[11]}$

São poucas evidências científicas que relatam a conduta do fisioterapeuta em ambiente hospitalar. De acordo com os estudos de Newstead et al 2017 e Lottering e Aswegen 2016, a conduta fisioterápica respiratória ainda é muito utilizada e corrobora com evidências encontradas neste trabalho, como por exemplo a utilização de exercícios de respiração profunda e da vibratoterapia e a pouca ou quase nula utilização do flutter como técnica de higiene brônquica. 
No entanto, outros estudos comprovam a heterogeneidade das técnicas utilizadas por fisioterapeutas em ambiente hospitalar. Observa-se que ainda há utilização de técnicas como a tapotagem, drenagem postural e inspirômetro incentivador, pouco utilizados ou não utilizados nos atendimentos realizados neste estudo. ${ }^{[7,14,15]}$

$\mathrm{Na}$ atualidade, a fisioterapia no Brasil está intimamente relacionada a prática da mobilização precoce. Estudo comprova que a mobilização precoce resulta em menor tempo de internação hospitalar e mantém níveis de funcionalidade aproximado ao normal, outro fator importante é a melhora da qualidade de vida pós-alta hospitalar ${ }^{[16]}$. Dessa forma, observamos em nosso estudo, predomínio do uso da mobilização ativa, seguida de deambulação e mobilização ativa-assistida, com utilização de mobilização passiva superior à resistida, sendo relevante ressaltar que existem barreiras e facilitadores para realização da mobilização precoce que devem ser avaliados pelo fisioterapeuta. ${ }^{[17]}$

\section{CONCLUSÃO}

Diante do exposto, conclui-se que o perfil dos pacientes encaminhados para fisioterapia são na maioria homens idosos, nas áreas de oncologia, gastroenterologia e cardiologia, com presença de múltiplos fatores de risco. Além disso, o as técnicas empregadas com maior frequência pela equipe de fisioterapia, dependem da participação ativa do paciente, o que nos permite concluir a importância do fisioterapeuta em unidades hospitalares, dado o seu perfil incentivador à promoção de atividades que possam reestabelecer a funcionalidade dos pacientes.

\section{REFERÊNCIAS}

1- $\quad$ Scherer MDA, Conill EM, Jean R, Taleb A, Gelbcke FL, Pires DEP, Joazeiro EMG. Desafios para o trabalho em saúde: um estudo comparado de Hospitais Universitários na Argélia, Brasil e França. Ciênc. Saúde coletiva vol.23 no.7, 2265-2276, Rio de Janeiro. 2018.

2- $\quad$ DATASUS[internet]. Brasília(DF): Ministério da Saúde; 2005. [Citado em 07 de agosto de 2018]. Disponível em: http://tabnet.datasus.gov.br/cgi/tabcgi.exe?sih/cnv/niuf.def

3- $\quad$ Freitas FAZ, Santos ESS, Pereira LSM, Lustosa LP. Vulnerabilidade física de idosos na alta hospitalar. Fisioter. Pesqui. vol.24 no.3, 253-258, São Paulo July/Sept. 2017.

4- Oliveira GN, Vancini-Campanharo CR, Lopes MCBT, Barbosa DA, Okuno MFP, Batista REA. Correlação das categorias de classificação de risco com aspectos clínicos e desfechos. Rev. Latino-Am. Enfermagem vol.24, 1-9, Ribeirão Preto 2016.

5- Nozawa E, Sarmente GJV, Veja JM, Costa D, Silva JEP, Feltrim MI. Perfil de fisioterapeutas brasileiros que atuam em unidades de terapia intensiva. Fisioter. Pesqui. vol.15 no.2, 177-182, São Paulo 2008.

6- $\quad$ Agostini P, Reeve J, Dromard S, Singh S, Steyn RS, Naidu B. A survey of physiotherapeutic provision for patients undergoing thoracic surgery in the UK. Physiotherapy 99 (2013) 56-62. https://doi.org/10.1016/i.physio.2011.11.001 
7- Grammatopoulou E, Charmpas TN, Strati EG, Nikolaos MT, Evagelodimou A, Belimpasaki V, Skordilis EK. The scope of physiotherapy services provided in public ICUs in Greece: A pilot study, Physiotherapy Theory and 2017. https://doi.org/10.1080/09593985.2016.1266718

8- Santos JÁ, Braun C, Grande GHD, Pinto RZ, Padulla SAT. Características dos pacientes atendidos pela fisioterapia em um hospital público. Colloquium Vitae, vol. 7, n. Especial, Jul-Dez, 2015, p. 32-38.

9- $\quad$ Cordeiro P, Martins M. Mortalidade hospitalar em pacientes idosos no Sistema Único de Saúde, região Sudeste. Rev Saude Publica. 2018;52:69. https://doi.org/10.11606/S1518$\underline{8787.2018052000146}$

10- Teixeira JJ, Bastos GF, Souza AC. Perfil de internação de idosos. Rev Soc Bras Clin Med. 2017 jan-mar;15(1):15-20.

11- Poffo MR, Assis AV, Fracasso A, Londero Filho M, Alves SMM, Bald AP, Schmitt CB, Alves Filho NR. Perfil dos Pacientes Internados por Insuficiência Cardíaca em Hospital Terciário. International Journal of Cardiovascular Sciences. 2017;30(3):189-198.

12- Newstead CJ, Seaton JÁ, Johnston CL. Australian critical care nursing professionals' attitudes towards the use of traditional "chest physiotherapy" techniques. Hong Kong Physiotherapy Journal (2017) 36, 33-48. https://doi.org/10.1016/i.hkpj.2016.08.001

13- Lottering M, Aswegen H. Physiotherapy practice in South African intensive care units. SAJCC July 2016, Vol. 32, No. 1,11-16.

14- Ogawa KYL, Frigeri LB, Diniz JS, Ferreira CASA. Intervenção fisioterapêutica nas emergências cardiorrespiratórias. O Mundo da Saúde, São Paulo: 2009;33(4):457-466.

15- Bhat A, Chakravarthy K, Rao BK. Chest physiotherapy techniques in neurological intensive care units of India: A survey. Indian Journal of Critical Care Medicine June 201418 (6): 363-368. https://doi.org/10.4103/0972-5229.133890

16- Rocha MAR, Martinez BP, Silva MVZ, Forgiarini Junior LA . Early mobilization: Why, what for and how? / La movilización temprana: ¿Por qué, para qué y cómo? Med. intensiva; 41(7): 429-436, oct. 2017. https://doi.org/10.1016/i.medin.2016.10.003

17- Hodgson CL, Capell E, Tipping CJ. Early Mobilization of Patients in Intensive Care: Organization, Communication and Safety Factors that Influence Translation into Clinical Practice. Hodgson et al. Critical Care (2018) 22:77. https://doi.org/10.1186/s13054-018-1998-9 